\title{
Hemosiderotic dermatofibroma: Unique clinical presentation and dermoscopic findings of a rare dermatofibroma variant
}

David Surprenant, Karlee Novice, Michael Dreifke, George Garib, James Swan

Division of Dermatology, Loyola University Medical Center, Maywood, USA

Received: July 21, 2016

DOI: $10.5430 /$ crcp.v3n4p53

\author{
Accepted: August 18, 2016 \\ Online Published: September 1, 2016 \\ URL: http://dx.doi.org/10.5430/crcp.v3n4p53
}

\begin{abstract}
Hemosiderotic dermatofibromas (HDFs) are a rare variant of the relatively common dermatofibroma (DF). HDFs can clinically mimic melanoma as well as other melanocytic lesions and are diagnosed with histopathological examination. Histopathologic diagnosis is not straightforward as there are many DF variants and the features of several variants can often be found in one lesion. These lesions may represent a stage in development from common DF to aneurysmal dermatofibroma (ADF). This possible progression is important to recognize, as ADFs tend to exhibit increased tendency for local recurrence and can also be confused with malignant lesions. We present a case of a 65-year-old presenting with a rare HDF having unique clinical and dermatoscopic findings. Our case underscores the importance of recognizing this rare entity and stresses the importance of remaining mindful of the possible progression to $\mathrm{ADF}$, an entity with increased propensity for recurrence.
\end{abstract}

Key Words: Hemosiderotic dermatofibroma, Dermatofibroma

\section{INTRODUCTION}

Dermatofibromas (DFs) are very common benign fibrohistiocytic lesions. ${ }^{[1]}$ DFs typically appear as firm hard papules, plaques, or nodules with color varying from yellow to brown or purple. ${ }^{[2]}$ Most DFs are $0.5 \mathrm{~cm}$ to $2 \mathrm{~cm}$ in diameter and are commonly found on the limbs and trunk of adults. ${ }^{[3]}$ Lateral compression of these lesions may produce a characteristic depression, the so-called "dimple sign" associated with dermatofibromas. ${ }^{[2]}$ Under dermoscopy, typical features of DF include a central violaceous or reddish homogenous area with a central white scar-like patch and a peripheral light pigment network with variable vascular structures. ${ }^{[4,5]}$ Histologically, the lesion is composed of interlacing fascicles of spindled cells set within a loose collagenous stroma associated with epidermal changes. ${ }^{[1]}$ The lesional cells include a variable combination of fibroblasts, macrophages, and blood vessels. ${ }^{[1]}$ Several clinical and histopathological dermatofibroma variants have been reported in the literature. These variants include cellular, epitheloid, atypical, lipidized, clear cell, palisading, atrophic, keloidal, granular cell, myxoid, lichenoid, balloon cell, signet-ring, hemosiderotic, and aneurysmal. ${ }^{[1]}$ The histologic features of several variants can coexist in the same lesion and in some cases may correspond to different stages of the same process. ${ }^{[6]}$ Hemosiderotic dermatofibroma (HDF) is a rare variant first described by Diss in 1938 as a lesion clinically mimicking melanoma. ${ }^{[6]}$ The differential diagnosis of a HDF comprises not only melanomas, but also other melanocytic and nonmelanocytic tumors. ${ }^{[2]}$

\footnotetext{
* Correspondence: David Surprenant, MD; Email: dsurpren@ gmail.com; Address: Loyola University Medical Center Division of Dermatology, 321 North La Grange Road, La Grange Park, IL 60526, USA.
} 
The diagnosis is made via histopathological examination. We report a unique case of a 65 -year-old gentleman who presented with a HDF overlying the Achilles tendon with an unexpected clinical presentation and interesting histopathology.

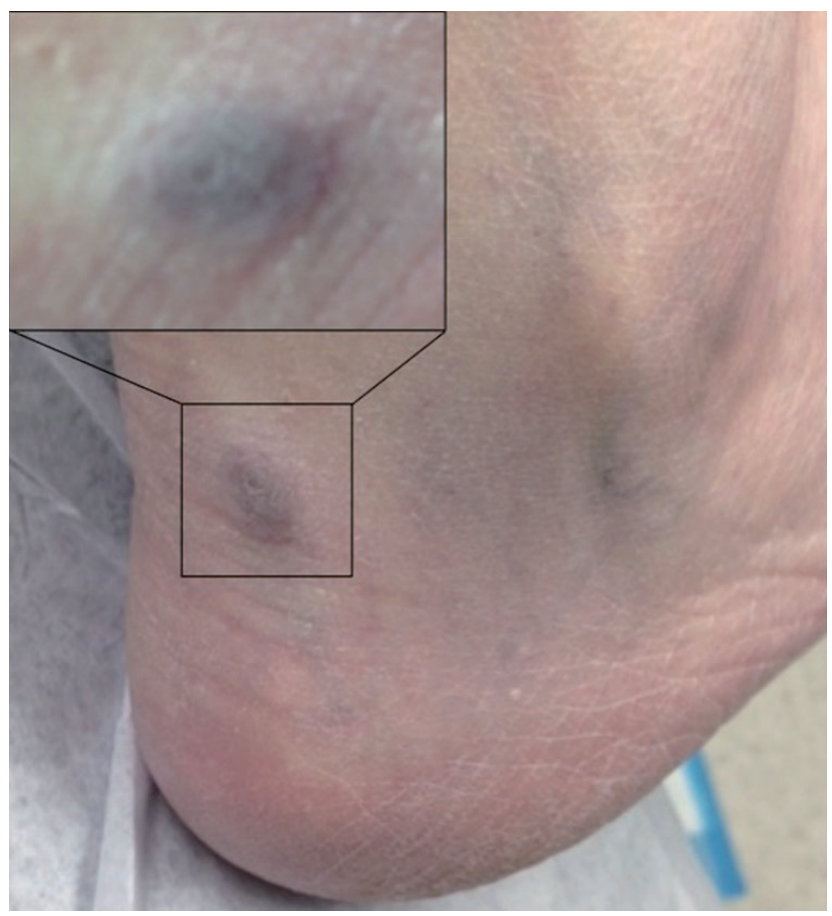

Figure 1. Well circumscribed, $1 \mathrm{~cm} \times 1 \mathrm{~cm}$, symmetric and uniform violaceous nodule with regular borders located on the right Achilles tendon

\section{CASE REPort}

A 65-year-old Caucasian male with no personal or family history of skin cancer presented for evaluation of a growth over his Achilles tendon for the past 6 months. The lesion was mildly tender with pressure but otherwise asymptomatic. Located on the right Achilles tendon was a well circumscribed, $1 \mathrm{~cm} \times 1 \mathrm{~cm}$, symmetric and uniform violaceous nodule with regular borders (see Figure 1). Our differential diagnosis included glomus tumor, malignant melanoma, venous lake, and dermatofibroma. Dermoscopy revealed a white central patch with no evidence of pigment network. A punch biopsy gave way easily, indicating a soft texture. Although the biopsy was partially disrupted, histopathological analysis of the visualized areas revealed a well circumscribed dermal spindle cell tumor with individual hyaline collagen bundles surrounded by tumor cells in the periphery of the lesion (see Figure 2). The tumor was composed of interlacing fascicles of spindled cells, foamy histiocytes, hemorrhagic irregular cleft-like and cystic spaces lacking endothelial lining and admixed hemosiderin deposition (see Figure 3). Immunohistochemical stains performed included CD68 which showed strong focal positivity in the histiocytes (see Figure 4). Since the differential diagnosis included vascular and melanocytic tumors, CD34 and S100 were performed. CD34 highlighted some scattered vessels while S-100 was negative in the cells of interest. Additional negative immunohistochemical stains included smooth muscle actin (SMA), cytokeratin AE1/AE3, and Factor XIIIa. The histopathological and immunohistochemical findings were most consistent with a HDF.

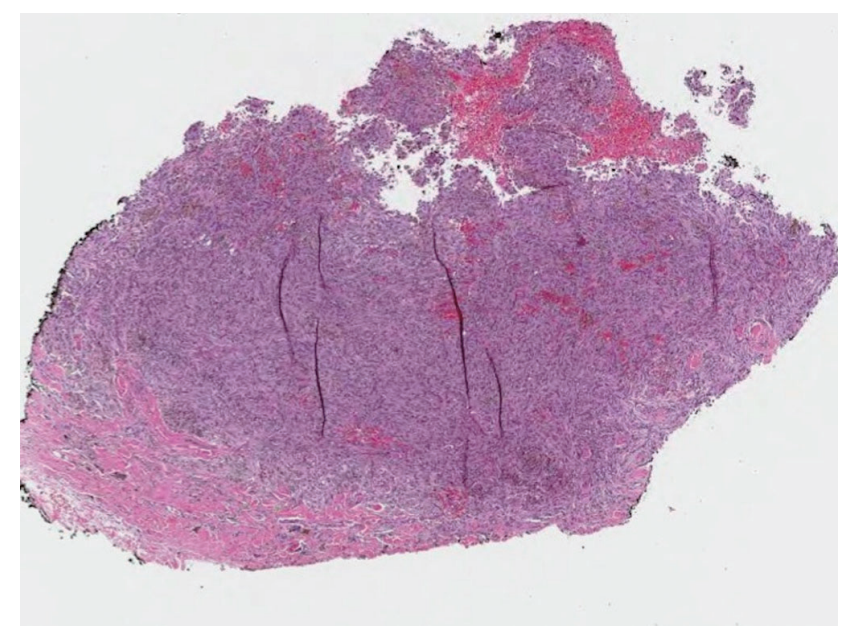

Figure 2. Punch biopsy shows a well circumscribed dermal spindle cell tumor with individual hyaline collagen bundles surrounded by tumor cells in the periphery of the lesion ("collagen trapping"). In addition, significant hemorrhage and irregular cleft-like spaces are noted. $(\mathrm{H} \& \mathrm{E} \times 2)$

\section{Discussion}

DFs are generally considered benign with less than $2 \%$ recurrence, often due to incomplete excision. ${ }^{[8]}$ Several DF variants exist and are distinguished by their histopathological characteristics. ${ }^{[8]}$ However, variant characterization is not always clear-cut, as the histologic features of several variants can be present within the same lesion. ${ }^{[2]}$

HDFs are a rare tumor; ${ }^{[4]}$ Alves et al. reviewed 192 DFs diagnosed in 181 patients and found that $5.7 \%$ represented the HDF variant. ${ }^{[1]}$ The true incidence is most likely much less considering that most DFs are not excised. HDFs commonly present as an asymptomatic, blue-violaceous, smooth surfaced nodule. ${ }^{[1]}$ They are dermoscopically described as having a central violaceous or reddish homogenous area with a central white scar-like patch and a peripheral light pigment network with variable vascular structures. ${ }^{[4]}$ The differential diagnosis includes melanoma and nonmelanocytic tumors, such as Spitz nevus, dermal nevus, targetoid hemosiderotic nevus, targetoid hemosiderotic hemangioma, and Kaposi sarcoma. ${ }^{[1]}$ The diagnosis is made histologically with findings of numerous small vessels, extravasated erythrocytes, and 
intra- and extracellular hemosiderin deposition due to hemorrhage. ${ }^{[1]}$ Our patient's case was unique given the lack of pigment network noted on dermoscopy as well as the soft texture on exam.

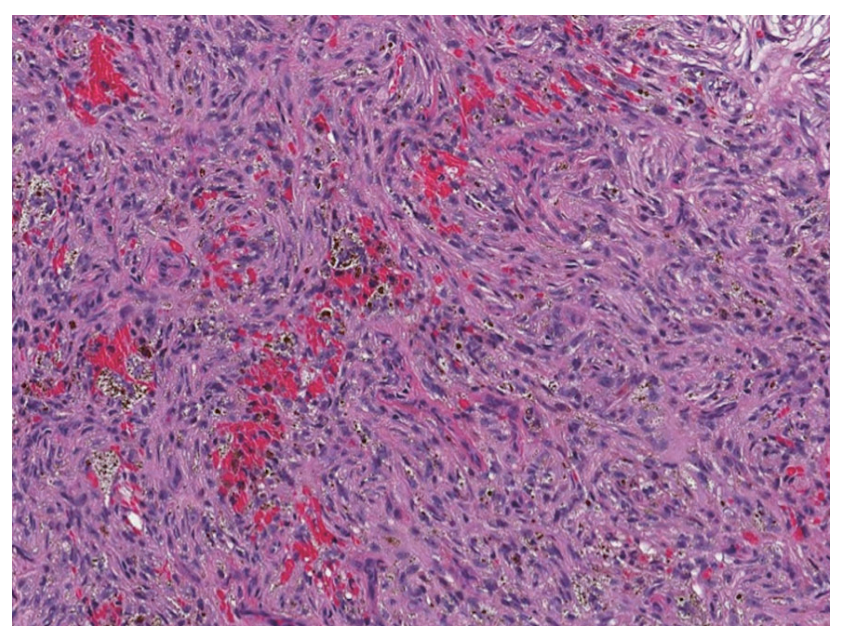

Figure 3. The tumor is composed of interlacing fascicles of spindled cells, foamy histiocytes, hemorrhagic irregular cleft-like and cystic spaces lacking endothelial lining and admixed hemosiderin deposition $(\mathrm{H} \& \mathrm{E} \times 20)$

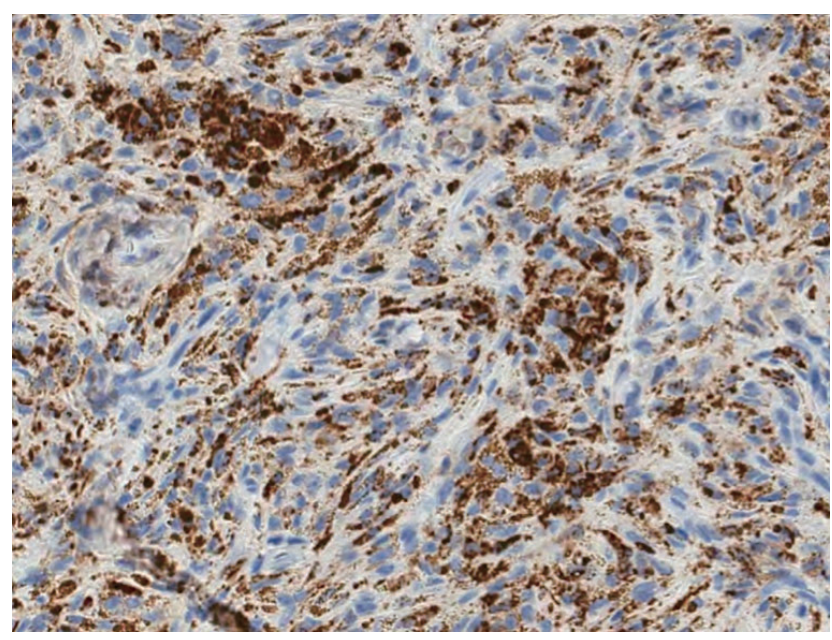

Figure 4. Immunohistochemical stain CD68 shows strong focal positivity in the histiocytes $(\mathrm{CD} 68 \times 40)$

Several published reports state that HDFs may represent a stage in development of aneurysmal dermatofibromas $(\mathrm{ADFs})^{[2,3]}$ and many authors consider HDF to be a precursor stage in the formation of ADF. ${ }^{[6]}$ Santa Cruz and
Kyriakos first described ADF as a clinically and histologically distinct entity. ${ }^{[7]}$ Individuals typically first develop ADFs during their thirties. These lesions are found more frequently in females than males. ${ }^{[8]}$ The lesion represents $1.7 \%$ of all DFs and often present as a solitary papule of variegated color ranging in size from $5 \mathrm{~mm}$ to $40 \mathrm{~mm} .{ }^{\left[{ }^{8]}\right.}$ Sudden growth of a previously stable lesion is frequently observed, secondary to hemorrhage. Histologically, ADF is an ill-defined lesion predominantly located in the dermis, occasionally observed extending into superficial fatty tissue, often with a variable degree of epidermal acanthosis. A defining histological feature of ADF, beyond the features seen in common DFs, are blood filled spaces similar to those seen in cavernous hemangiomas. These blood filled spaces lack an endothelial lining and are instead lined by cells typical of a DF - histiocytes, siderophages, foam cells, fibroblasts, and occasionally, giant cells. Surrounding stroma contains abundant small capillaries with prominent interstitial hemorrhage and hemosiderin deposition. In contrast to common DFs, which recur less than $2 \%$ of the time, recurrences following incomplete excision of $\mathrm{ADF}$ have been reported to be up to $19 \% .^{[8]}$

Authors that believe that HDFs exist as a precursor to ADFs postulate that in some DFs, small amounts of blood slowly extravasate from capillaries within the tumor, resulting in hemosiderin deposition. ${ }^{[6]}$ It is hypothesized that tumor cells subsequently phagocytize this pigment, resulting in the HDF variant. Continued slow blood leak may account for the final formation of the characteristic blood filled spaces of ADFs. ${ }^{[2]}$ The evolution from a hemosiderotic DF to an aneurysmal DF is supported by dermoscopic-histopathological correlation. ${ }^{[2]}$

It is important to recognize HDF and ADF as DF variants as they can be incorrectly diagnosed as malignancies. ${ }^{[6]}$ In addition, although generally considered a benign tumor, the cellular, aneurysmal, and atypical variants of cutaneous DFs have a significant tendency for local recurrence. ${ }^{[9]}$ Overall, we report a case of a unique presentation of a HDF and emphasize the importance of differentiating a HDF from other tumors and malignancies while underscoring the possibility of conversion to a variant, such as $\mathrm{ADF}$, with higher rates of local recurrence.

\section{CONFLicts OF INTEREST Disclosure}

The authors declare no conflicts of interest. 


\section{REFERENCES}

[1] Alves JVP, Matos DM, Barreiros HF, et al. Variants of dermatofibroma-a histopathological study. An Bras Dermatol. 2014; 3: 472-7. http://dx.doi.org/10.1590/abd1806-4841.20142629

[2] Scalvenzi M, Balato A, De Natale F, et al. Hemosiderotic Dermatofibroma: Report of One Case. Dermatology. 2007; 214: 82-84. PMid: 17191053. http://dx.doi.org/10.1159/000096918

[3] Pusztaszeri M, Jaquet PY, Williamson C. Giant Hemosiderotic Dermatofibroma: A Case Report and Review of the Literature. Case Rep Dermatol. 2011; 3: 32-6. PMid: 21487458. http://dx.doi.org $/ 10.1159 / 000324721$

[4] Blum A, Jaworski S, Metzler G, et al. Lessons on Dermoscopy: Dermoscopic Pattern of Hemosiderotic Dermatofibroma. Dermatol Surg. 2004; 30: 1354-5. http://dx.doi.org/10.1097/00042728-2 00410000-00014

[5] Ferrari A, Soyer HP, Peris K, et al. Central white scarlike patch: a dermatoscopic clue for diagnosis of dermatofibroma. J Am Acad
Dermatol. 2000; 43: 1123-5. http://dx.doi.org/10.1067/mjd .2000 .109842

[6] Zaballos P, Llambrich A, Ara M, et al. Dermoscopic findings of haemosiderotic and aneurysmal dermatofibroma: report of six paitents. British Journal of Dermatology. 2006; 154: 244-50. PMid: 16433792. http://dx.doi.org/10.1111/j.1365-2133. 2005 $.06844 . \mathrm{x}$

[7] Cruz DJ, Kyriakos M. Aneurysmal ("angiomatoid") fibrous histiocytoma of the skin. Cancer. 1981; 47(8): 2053-61. http://dx.doi.org/10.1002/1097-0142(19810415)47: 8<2053: : AID-CNCR2820470825>3.0.CO;2-A

[8] Luzar B, Calonje E. Cutaneous fibrohistiocytic tumours-an update. Histopathology. 2010: 56; 148-65. PMid: 20055912. http: //dx.doi.org/10.1111/j.1365-2559.2009.03447.x

[9] Dole L, Fletcher C. Metastasizing "Benign" Cutaneous Fibrous Histiocytoma. Am J Surg Path. 2013; 37: 484-95. PMid: 23426120. http://dx.doi.org/10.1097/PAS.0b013e31827070d4 